\title{
Branched-chain amino acid aminotransferase 2 regulates ferroptotic cell death in cancer cells
}

\author{
Kang Wang ${ }^{1} \cdot$ Zhengyang Zhang $^{1} \cdot$ Hsiang-i Tsai ${ }^{1} \cdot$ Yanfang Liu $^{2} \cdot$ Jie Gao ${ }^{1} \cdot$ Ming Wang $^{1} \cdot$ Lian Song $^{1} \cdot$

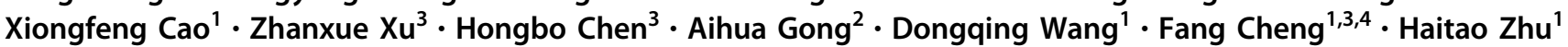

Received: 22 March 2020 / Revised: 9 October 2020 / Accepted: 12 October 2020 / Published online: 23 October 2020

(c) The Author(s) 2020. This article is published with open access

\begin{abstract}
Ferroptosis, a form of iron-dependent cell death driven by cellular metabolism and iron-dependent lipid peroxidation, has been implicated as a tumor-suppressor function for cancer therapy. Recent advance revealed that the sensitivity to ferroptosis is tightly linked to numerous biological processes, including metabolism of amino acid and the biosynthesis of glutathione. Here, by using a high-throughput CRISPR/Cas9-based genetic screen in HepG2 hepatocellular carcinoma cells to search for metabolic proteins inhibiting ferroptosis, we identified a branched-chain amino acid aminotransferase 2 (BCAT2) as a novel suppressor of ferroptosis. Mechanistically, ferroptosis inducers (erastin, sorafenib, and sulfasalazine) activated AMPK/ SREBP1 signaling pathway through iron-dependent ferritinophagy, which in turn inhibited BCAT2 transcription. We further confirmed that BCAT2 as the key enzyme mediating the metabolism of sulfur amino acid, regulated intracellular glutamate level, whose activation by ectopic expression specifically antagonize system $\mathrm{Xc}^{-}$inhibition and protected liver and pancreatic cancer cells from ferroptosis in vitro and in vivo. On the contrary, direct inhibition of BCAT2 by RNA interference, or indirect inhibition by blocking system $\mathrm{Xc}^{-}$activity, triggers ferroptosis. Finally, our results demonstrate the synergistic effect of sorafenib and sulfasalazine in downregulating BCAT2 expression and dictating ferroptotic death, where BCAT2 can also be used to predict the responsiveness of cancer cells to ferroptosis-inducing therapies. Collectively, these findings identify a novel role of BCAT2 in ferroptosis, suggesting a potential therapeutic strategy for overcoming sorafenib resistance.
\end{abstract}

These authors contributed equally: Kang Wang, Zhengyang Zhang, Hsiang-i Tsai

Edited by S. Fulda

Supplementary information The online version of this article (https:// doi.org/10.1038/s41418-020-00644-4) contains supplementary material, which is available to authorized users.

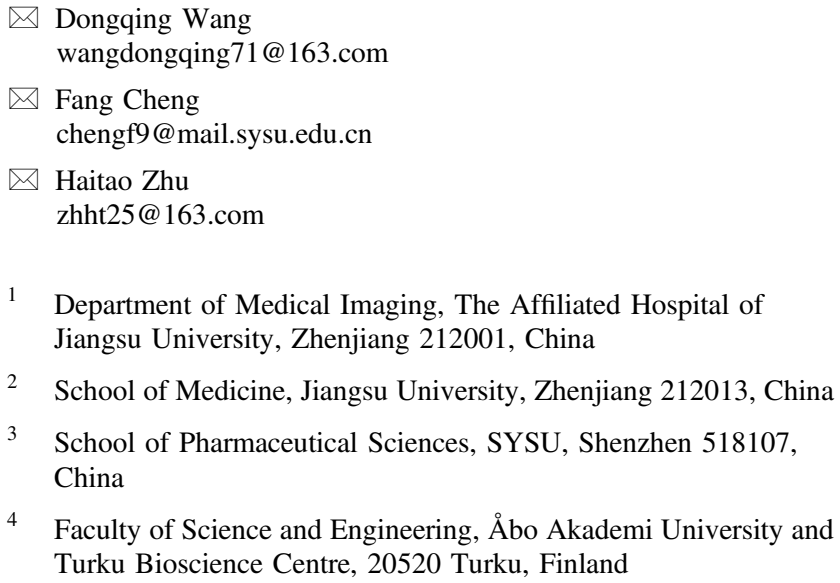

1 Department of Medical Imaging, The Affiliated Hospital of Jiangsu University, Zhenjiang 212001, China

2 School of Medicine, Jiangsu University, Zhenjiang 212013, China

3 School of Pharmaceutical Sciences, SYSU, Shenzhen 518107, China

4 Faculty of Science and Engineering, Åbo Akademi University and Turku Bioscience Centre, 20520 Turku, Finland

\section{Introduction}

Ferroptosis is emerging as an iron-dependent type of regulated cell death, which is induced by the loss of cellular redox homeostasis, leading to unchecked lipid peroxidation and eventually cell death [1]. Pharmacological inactivation of cystine-glutamate antiporter (system $\mathrm{Xc}^{-}$) or glutathione peroxidase 4 (GPX4) can induce ferroptosis [2] suggesting the crucial roles of the glutathione-dependent antioxidant defenses in preventing ferroptotic cell death.

Ferroptosis has been implicated in ischemia-induced organ injury, pathological cell death associated with degenerative diseases, and in different types of cancer [3-5]. A variety of tumor cells are susceptible to ferroptosis including lymphoma, cervical cancer, head and neck cancer, pancreatic cancer, renal cell carcinoma, and hepatocellular carcinoma (HCC). Various studies have confirmed the pivotal role of ferroptosis inducers, including small-molecule ferroptosis inducers such as erastin as well as a number of drugs (e.g., sorafenib, artemisinin, and its derivatives) in killing tumor cells and suppressing tumor 
growth [6-8]. These ferroptosis inducers could also synergy with chemotherapeutic drugs in cancer treatment. Interestingly, some types of cancer are more sensitive to ferroptosis inducers than others. The reverse transsulfuration pathway has been identified as a negative regulator of ferroptosis, and a deficiency of this pathway in ovarian cancer cells is associated with increased sensitivity to erastin-induced ferroptosis [9]. HSF1-HSPB1 pathway also negatively regulated erastininduced ferroptosis in human cervical cancer, prostate cancer, and osteosarcoma [10]. MUC1-C/xCT pathway is another negative regulator in erastin-induced ferroptosis of triplenegative breast cancer cells [11].

Accumulating evidence indicates that cellular metabolism plays a crucial role in ferroptosis [12, 13]. The transcriptional factor NRF2 coordinates the antioxidant defensive system in the regulation of ferroptosis. The p62Keap1-NRF2 is a central inhibitory pathway of ferroptosis in liver cancer cells [14]. Genetic or pharmacologically inhibition of NRF2 significantly enhanced ferroptosis susceptibility of liver cancer induced by erastin and sorafenib, whereas the activation of NRF2 expression leds to cellular resistance to ferroptosis, suggesting a central role of the partially reduced oxygen-containing molecules, especially reactive oxygen species (ROS) in ferroptosis. Intracellular iron metabolism is also essential for ferroptosis through either the action of iron-dependent oxidases, or by Fenton chemistry. A recent report suggests that autophagic degradation of ferritin regulates ferroptosis through an autophagy cargo receptor nuclear receptor coactivator 4 (NCOA4) [15]. Not surprisingly, amino acid metabolism is also involved in ferroptosis [16, 17]. High concentration of extracellular glutamate, erastin, or other system $\mathrm{Xc}^{-}$inhibitors block intracellular cystine/ cysteine uptake to induce ferroptosis. Silencing cysteinyltRNA synthetase (CARS) upregulates the transsulfuration pathway, which leads to resistance to erastin-induced ferroptosis. Glutamine mediates ferroptosis through its specific metabolic enzymes, glutaminases (GLS1 and GLS2), though the mechanism of glutaminolysis process is complex. However, the metabolic pathways controlling ferroptosis sensitivity in liver cancer cells remains unclear.

In this study, we identify a branched-chain amino acid aminotransferase 2 (BCAT2), an aminotransferase enzymemediating sulfur amino acid metabolism, as a specific inhibitor of ferroptosis. We show that BCAT2 is involved in system $\mathrm{Xc}^{-}$inhibitor-induced ferroptosis in liver cancer cells. Furthermore, BCAT2 participates in the mechanisms for sulfasalazine synergizing with sorafenib to induce ferroptosis. Thus, our results demonstrate that BCAT2 serves as a suppressor of ferroptosis, and contributes to the core metabolic signaling pathways involved in liver cancer ferroptosis.

\section{Results}

\section{Identification of novel players of ferroptosis by kinome CRISPRa screening}

Ferroptosis can be induced by two classes of smallmolecule substances known as class 1 system $\mathrm{Xc}^{-}$inhibitors (including erastin, sulfasalazine, and sorafenib) and class 2 ferroptosis inducers (GPX4 inhibitors). We first tested and confirmed the effects of erastin (Erastin), sorafenib (SOR), and sulfasalazine (SAS) as probes to induce ferroptosis in human pancreatic cancer cell line Aspc-1, human HCC cell line HepG2, human colorectal cancer cell line SW480, as well as human fibrosarcoma cell line HT1080. The results confirmed that erastin, sorafenib, and sulfasalazine could significantly induce the cancer cell death at the concentration of $10 \mu \mathrm{mol} / \mathrm{L}$ for erastin, 5 $\mu \mathrm{mol} / \mathrm{L}$ for sorafenib, and $1 \mathrm{mmol} / \mathrm{L}$ for sulfasalazine, respectively (Figs. S1A and S1B). Furthermore, the cell death was dramatically inhibited by ferroptosis inhibitor ferrostatin-1, but not by apoptotic inhibitor ZAVD-FMK or necroptosis inhibitor Necrosulfonamide, indicating the specificity of all three ferroptosis inducers (Figs. S1A and S1B).

To systemically elucidate conserved downstream negative regulators of ferroptosis, we performed a large-scale genetic CRISPR activation (CRISPRa) screen. A pooled human CRISPRa sgRNA lentivirus library targeting 2320 Kinases, Phosphatases, and Drug Targets (https://www.addgene.org/ pooled-library/weissman-human-crispra-v2-subpools/) together with Cas9-VPR enzyme were introduced into HepG2 cells by lentiviral transduction, which were then treated with erastin or control DMSO (Fig. 1A). Deep sequencing of the sgRNAs integrated into genomic DNA from control cells and cells that survived ferroptosis induction was subsequently performed. Comparison of the sequencing data leds to the identification of $\operatorname{sgRNAs}$ that were enriched in cells surviving ferroptosis treatment. The gene targets of the enriched sgRNAs are potential genes that confer resistance to erastinmediated ferroptosis in HepG2 cells. Among the screen hits, many reported ferroptosis genes were identified and validated in our screen approach. Intriguingly, our screen also identified genes not previously implicated in the regulation of ferroptosis, among which branched BCAT2 was the top candidate of potential negative regulators in ferroptosis (Fig. 1A).

Branched-chain amino acid aminotransferase (BCAT) is an aminotransferase enzyme, which acts upon branchedchain amino acids (BCAAs) to regulate sulfur amino acid metabolism. To validate the role of BCAT2 in ferroptosis, we first investigated the expression of BCAT2 in above mentioned four cancer cell lines upon induction of erastin, sorafenib, and sulfasalazine. Western blot and quatitive real-time polymerase chain reaction (qRT-PCR) showed 
A

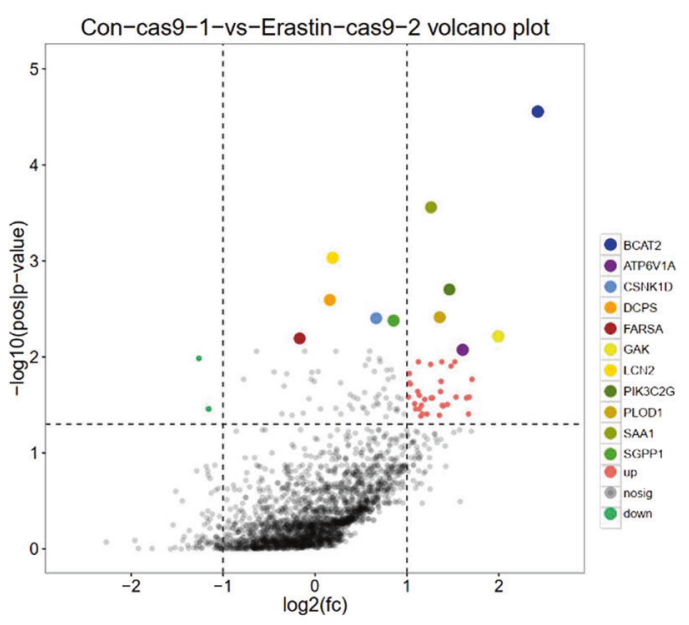

B

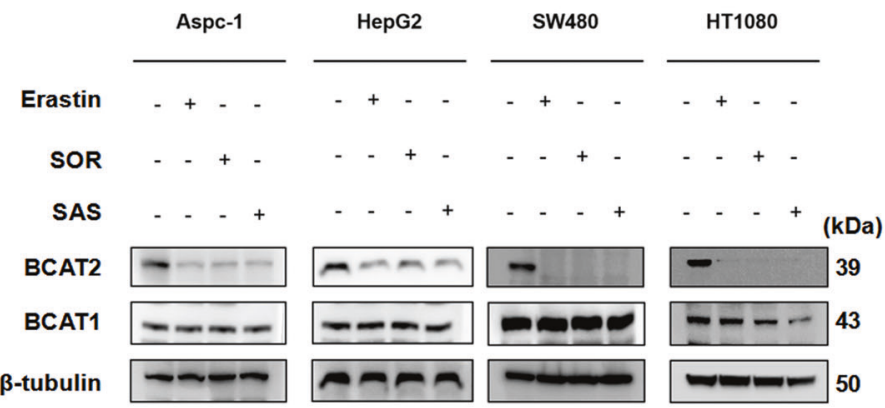

C
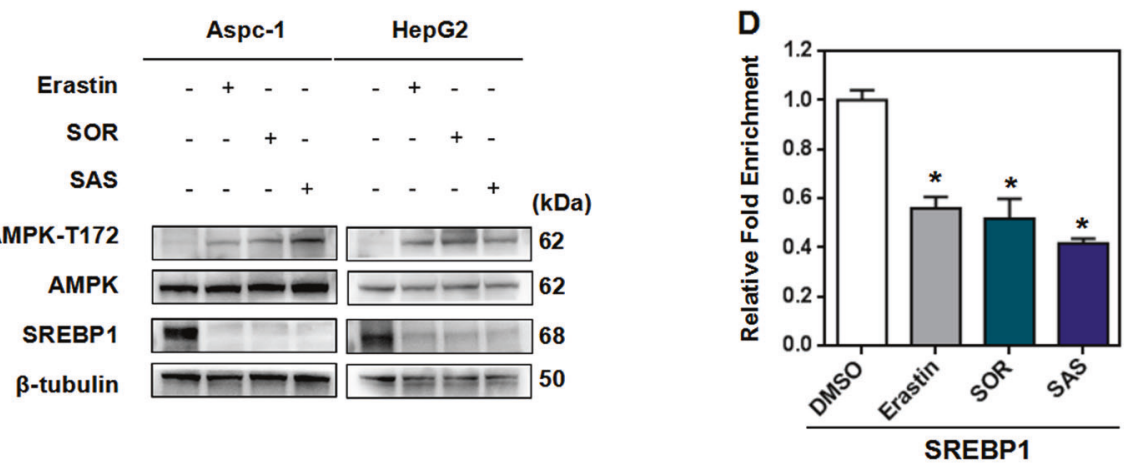

E
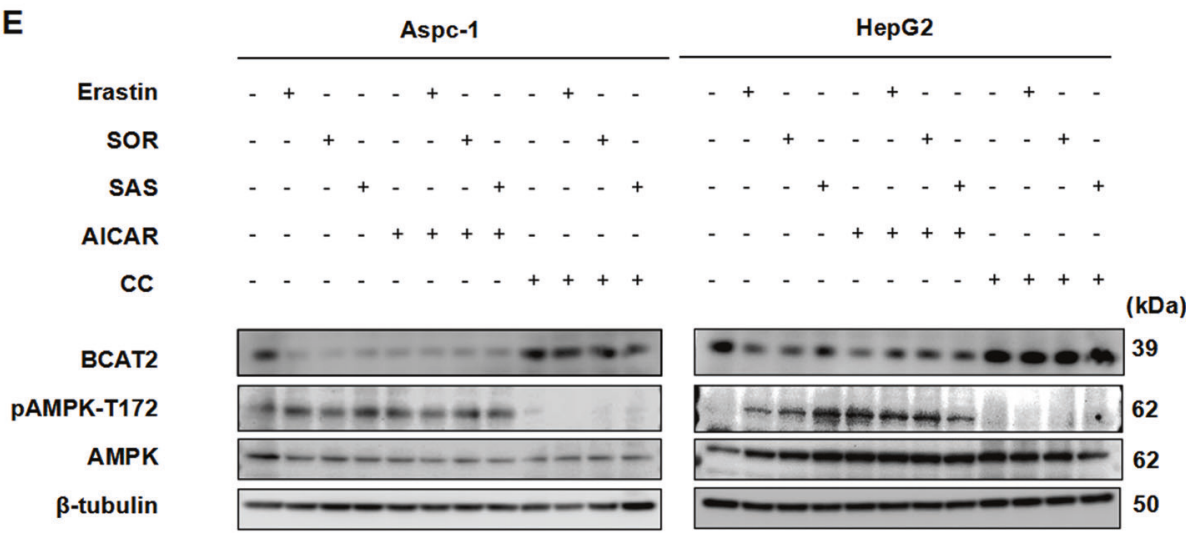

that in all four cell lines, there was a reduction of BCAT2, but not BCAT1 (a paralog of BCAT2) in proteinand mRNA-expression levels upon ferroptosis inducer treatment, which was reversed in the presence of deferoxamine mesylate (DFO, a ferroptosis inhibitor) (Figs. 1B, S2A, S2B, and S7). Moreover, erastin, sorafenib, and 
Fig. 1 Ferroptosis inducers inhibit BCAT2 expression through ferrtinophagy-AMPK-SREBP1 pathway. A A large-scale genetic CRISPR activation (CRISPRa) screen identifies genes essential for regulating ferroptosis. HepG2 cells expressing dcas9 were mutagenized with a pooled lentiviral sgRNA library. Significant hits from screens in cells treated with erastin or DMSO-treated cells. Dots represent individual genes. Colorful dots indicate significant enrichment genes that are resistant to erastin-mediated ferroptosis. $X$ axis indicated the fold change of gRNA insertions per gene (treatment group over control group), $Y$ axis represents the frequency of insertions $(p<0.05)$. B Western blot analysis of the protein expression levels of BCAT2 and BCAT1 in Aspc-1, HepG2, SW480 and HT1080 cells treated with DMSO (control), erastin $(20$ or $10 \mu \mathrm{mol} / \mathrm{L})$, sorafenib(10 or $5 \mu \mathrm{mol} / \mathrm{L})$, or sulfasalazine $(2$ or $1 \mathrm{mmol} / \mathrm{L})$. $\beta$-tubulin expression was detected as a loading control. C Western blot analysis of the protein expression levels of AMPK, pAMPK(T172), and SREBP1 in Aspc-1 and HepG2 cells treated with DMSO (control), erastin $(20$ or $10 \mu \mathrm{mol} / \mathrm{L})$, sorafenib ( 10 or $5 \mu \mathrm{mol} / \mathrm{L}$ ), or sulfasalazine ( 2 or $1 \mathrm{mmol} / \mathrm{L}$ ). $\beta$-tubulin expression was detected as a loading control. D Chromatin immunoprecipitation (ChIP) analysis of SREBP1 binding to BCAT2 promoter in HepG2 cells treated with DMSO (control), erastin $(20 \mu \mathrm{mol} / \mathrm{L})$, sorafenib $(10 \mu \mathrm{mol} / \mathrm{L})$, or sulfasalazine $(2 \mathrm{mmol} / \mathrm{L})$. E Western blot analysis of the protein expression levels of BCAT2, AMPK, and pAMPK(T172) in Aspc-1, and HepG2 cells treated with DMSO (control), erastin (20 or $10 \mu \mathrm{mol} / \mathrm{L})$, sorafenib $(10$ or $5 \mu \mathrm{mol} / \mathrm{L})$, or sulfasalazine ( 2 or $1 \mathrm{mmol} / \mathrm{L}$ ) in the absence or presence of AICAR (AMPK activator, $2 \mathrm{mmol} / \mathrm{L}$ ) and Compound C (AMPK inhibitor, $1 \mu \mathrm{mol} / \mathrm{L}$ ). $\beta$-tubulin expression was detected as a loading control. AMPK represents for AMP-activated protein kinase; SREBP1 represents for sterol response element binding protein 1; pAMPK-T172 represents for AMPK phosphorylation on threonine residue 172 (T172); CC represents Compound C; SOR represents sorafenib; SAS represents sulfasalazine. Experiments were repeated three times, and the data are expressed as the mean \pm SEM. $* p<0.05$ vs. control group. Statistical analysis was performed using Student's $t$ test.

sulfasalazine downregulated the BCAT2 protein level in a time-dependent manner (Fig. S2C). The Cancer Genome Atlas (TCGA) data analysis showed that BCAT2 expression correlated with cancer grade (Fig. S3A) and the expression of ferroptosis markers (GPX4 and NCOA4) in HCC (Fig. S3B).

\section{Ferroptosis inducers inhibit BCAT2 expression through ferritinophagy-AMPK-SREBP1 pathway}

It has been reported that AMP-activated protein kinase (AMPK) inhibits nuclear translocation of sterol response element binding protein 1 (SREBP1), which consequently suppresses the transcription of its direct target gene BCAT2 [18]. Therefore, we hypothesize that ferroptosis inducers downregulate BCAT2 via AMPK-SREBP1 signaling pathway. We confirmed that erastin, sorafenib, or sulfasalazine induced AMPK phosphorylation on threonine residue 172 (T172) and reduced the expression of SREBP1, assessed by quantifying the signals from western blotting (Figs. 1C, S4A, and S4B). ChIP assay also revealed that SREBP1 binding to BCAT2 was significantly reduced in the presence of erastin, sorafenib, or sulfasalazine (Fig. 1D), suggesting that they further prevent the transcription factor SREBP1 to activate BCAT2 transcription in the nucleus. Moreover, AMPK activator AICAR downregulated BCAT2 expression in both Aspc-1 and HepG2 cancer cells in a similar manner to erastin, sorafenib, or sulfasalazine, which can be reversed by AMPK inhibitor Compound $\mathrm{C}$, further confirming that ferroptosis inducers downregulate BCAT2 expression through activating AMPK (Figs. 1E, S4C, and S4D). We next further explore the relationship between SREBP1 and BCAT2 in both SREBP1 knockout (Fig. S5A) and knockdown HepG2 cancer cells (Fig. S5B). SREBP1 knockout HepG2 cancer cells using CRISPR-Cas9 technology displayed the down-expression of BCAT2 (Figs. S5C and S5D). Moreover, the expression level of BCAT2 was further reduced in the presence of erastin, sorafenib, or sulfasalazine in the SREBP1 knockout HepG2 cancer cells (Figs. S5C and S5D). The similar results can be acquired in the SREBP1 knockdown HepG2 cancer cells (Fig. S5E, F). Collectively, these results indicate that AMPK/ SREBP1 mediates BCAT2 expression in ferroptotic process.

Next, we would like to understand how AMPK/SREBP1 is activated by ferroptosis inducers. As AMPK is a promoter of autophagy and ferroptosis is an autophagic cell death process called ferritinophagy $[19,20]$, we asked whether ferritinophagy is involved in AMPK activity. NCOA4 was a selective cargo receptor for the selective autophagic turnover of ferritin [15]. First, we found that erastin, sorafenib, or sulfasalazine inhibited NCOA4 expression (Fig. S6A) and promoted the formation of GFP-LC3 puncta, the hallmarks of autophagy response (Fig. S6B), whereas inhibition of NCOA4 or autophagy can further decrease $\mathrm{Fe}^{2+}$ level (Fig. S6C-F). Moreover, erastin induced the AMPK phosphorylation on threonine residue 172 (T172), and BCAT2 inhibition can be reversed in the presence of BafA1 (an autophagy inhibitor) and DFO (an iron chelator) (Fig. S7), further indicating that AMPK/SREBP1 is the downstream of ferritinophagy and ferrous ions to inhibit BCAT2 expression. Therefore, erastin, sorafenib, or sulfasalazine inhibits BCAT2 expression through ferritinophagy-AMPKSREBP1 pathway.

\section{BCAT2 is a suppressor of ferroptotic cancer cell death}

To investigate the role of BCAT2 in ferroptosis, we first transfected BCAT2 cDNA plasmid into Aspc-1 and HepG2 cells and confirmed the overexpression of BCAT2 proteins in these cells by western blot (Fig. S8A). Given the critical role of iron in ferroptosis, we first examined the correlation of BCAT2 expression and iron accumulation. As expected, erastin, sorafenib, or sulfasalazine treatment induced freeiron accumulation in both control and BCAT2 transfected cells (Figs. 2A and S8B). Compared to the parental cells, overexpression of BCAT2 had no effect on the 
A

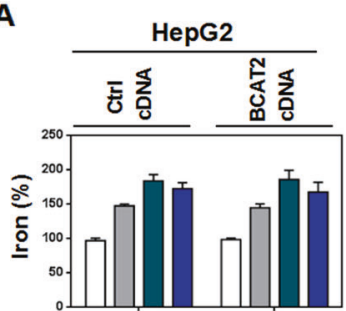

$\square$ DMSO $\square$ Erastin $\square$ SOR $\square$ sA

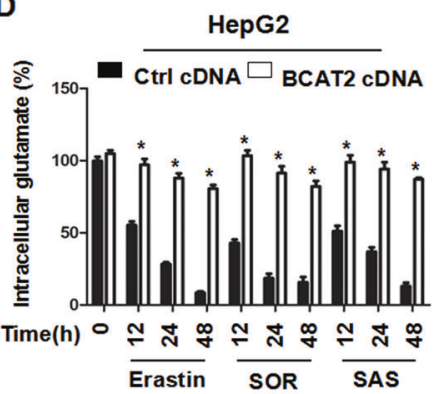

G

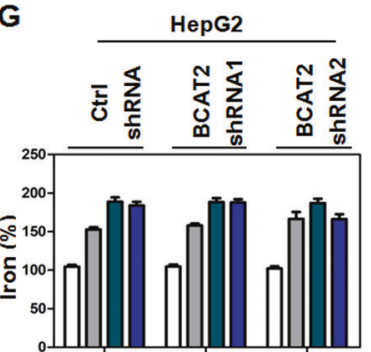

B

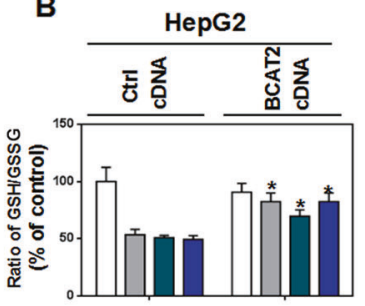

E

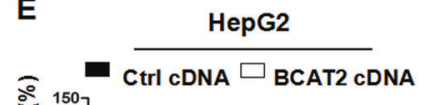

C

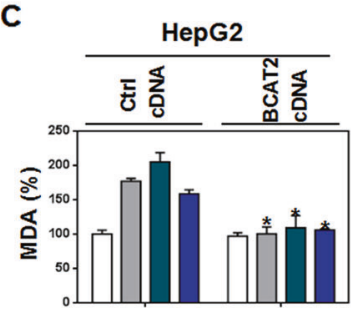

F

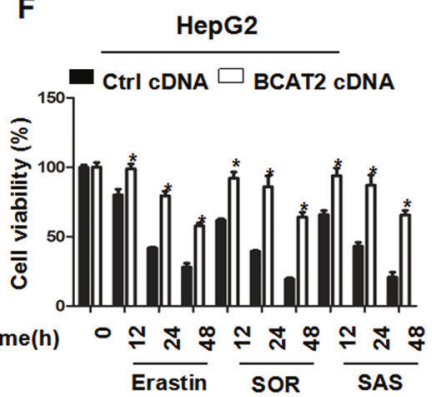

$\square$ DMSO $\square$ Erastin $\square$ SOR $\square$ SAS

J

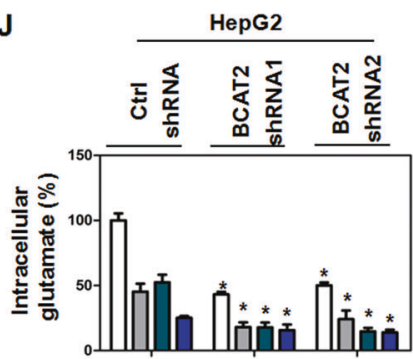

H

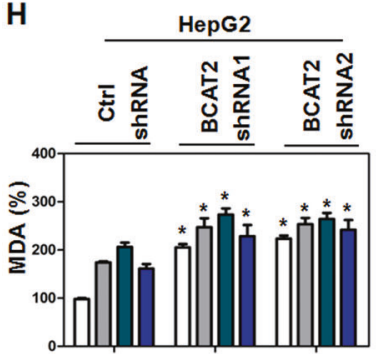

I
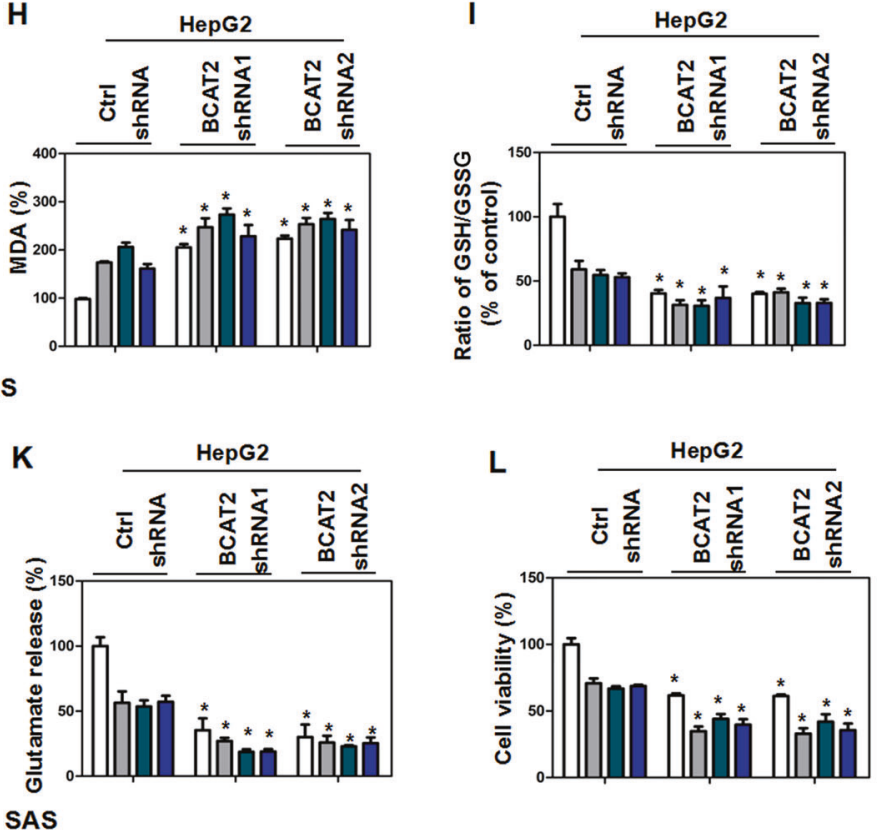

HepG2
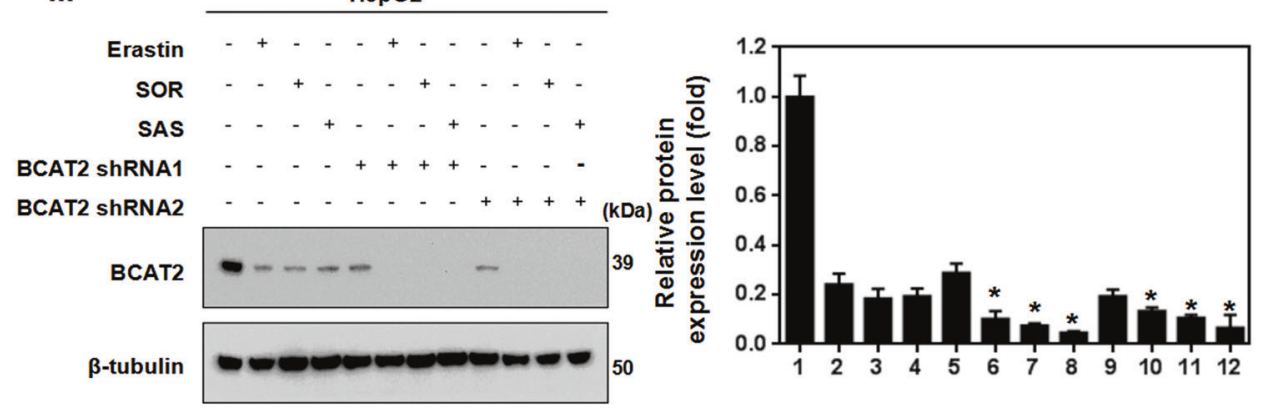

1 DMso

2 Erastin

3 SOR

4 SAS

5 DMSO + BCAT2 ShRNA1

6 Erastin + BCAT2 shRNA1

7 SOR + BCAT2 ShRNA1

8 SAS + BCAT2 ShRNA1

9 DMSO + BCAT2 shRNA2

10 Erastin + BCAT2 shRNA2

11 SOR + BCAT2 ShRNA2

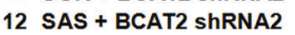

accumulation of $\mathrm{Fe}^{2+}$ in Aspc-1 and HepG2 cells following ferroptotic induction (Figs. 2A and S8B). Because system $\mathrm{Xc}^{-}$is responsible for maintaining redox homeostasis by importing cysteine to synthesize GSH, we asked whether BCAT2 is involved in system $\mathrm{Xc}^{-}$-mediated GSH activity. GSH level was inhibited in Aspc-1 and HepG2 cells 
Fig. 2 BCAT2 is a negative regulator of ferroptotic cancer cell death. A-C BCAT2 overexpressed and parental HepG2 cells were treated with DMSO (control), erastin $(20 \mu \mathrm{mol} / \mathrm{L})$, sorafenib $(10 \mu \mathrm{mol} / \mathrm{L})$, or sulfasalazine $(2 \mathrm{mmol} / \mathrm{L})$. The relative levels of $\mathrm{Fe}^{2+}(\mathbf{A})$, ratio of GSH/GSSG (B), and MDA (C) were assayed. D-F BCAT2 overexpressed and parental HepG2 cells were treated with DMSO (control), erastin $(20 \mu \mathrm{mol} / \mathrm{L})$, sorafenib $(10 \mu \mathrm{mol} / \mathrm{L})$, or sulfasalazine $(2 \mathrm{mmol} / \mathrm{L})$ for the indicated time $(0,12,24,48 \mathrm{~h})$. The relative levels of intracellular glutamate (D), glutamate release $(\mathbf{E})$, and cell viability $(\mathbf{F})$ were assayed. G-L BCAT2 knockdown and parental HepG2 cells were treated with DMSO (control), erastin $(20 \mu \mathrm{mol} / \mathrm{L})$, sorafenib $(10 \mu \mathrm{mol} / \mathrm{L})$, or sulfasalazine $(2 \mathrm{mmol} / \mathrm{L})$. The relative levels of $\mathrm{Fe}^{2+}(\mathbf{G})$, MDA $(\mathbf{H})$, ratio of GSH/GSSG (I), intracellular glutamate $(\mathbf{J})$, glutamate release $(\mathbf{K})$, and cell viability $(\mathbf{L})$ were assayed. $\mathbf{M}$ Western blot analysis of the protein expression levels of BCAT2 in BCAT2 knockdown and parental HepG2 cells treated with or without erastin $(20 \mu \mathrm{mol} / \mathrm{L})$, sorafenib $(10 \mu \mathrm{mol} / \mathrm{L})$, or sulfasalazine $(2 \mathrm{mmol} / \mathrm{L})$. $\beta$-tubulin expression was detected as a loading control. MDA represents malondialdehyde; GSH represents glutathione; GSSH represents oxidized glutathione; SOR represents sorafenib; SAS represents sulfasalazine. Experiments were repeated three times, and the data are expressed as the mean \pm SEM. ${ }^{*} p<0.05$ vs. control group. Statistical analysis was performed using Student's $t$ test.

following erastin, sorafenib, and sulfasalazine treatment, which was restored by ectopic expression of BCAT2 (Figs. 2B and S8C). The level of malondialdehyde (MDA), an end product of lipid peroxidation, was increased in Aspc1 and HepG2 cells following erastin, sorafenib, and sulfasalazine treatment, but decreased in BCAT2-overexpressing cells compared to their parental cells (Figs. 2C and S8D). In line with these results, in the presence of erastin, sorafenib, or sulfasalazine, BCAT2 overexpression increased intracellular glutamate (Figs. 2D and S8E) and the glutamate release (Figs. 2E and S8F), and reduced system $\mathrm{Xc}^{-}$ inhibitor-induced cell death (Figs. $2 \mathrm{~F}$ and $\mathrm{S} 8 \mathrm{G}$ ) in a timedependent manner. We also treated the parental and BCAT2 overexpression Aspc-1 and HepG2 cells with the other type ferroptosis inducer, RAS-selective lethal 3 (RSL3, GPX4 inhibitor), and BSO (GSH synthase inhibitor), and the similar results can be acquired (Fig. S9). Moreover, RSL3 and BSO had no effect on the expression level of BCAT2 in parental Aspc-1 and HepG2 cells (Fig. S10A). Due to the critical role of glutamine metabolism in ferroptosis, treated BCAT2 overexpression cancer cells with 6-diazo-5-oxo-Lnorleucine (DON, glutaminase inhibitor) could abolish the protective effect of BCAT2 in ferroptotic cancer cell death (Fig. S10B). In order to confirm the relationship between BCAT2 and ferroptosis in vivo, a subcutaneous xenograft tumor model was established by injecting $1 \times 10^{6}$ parental or overexpression BCAT2 Panc02 cancer cells into the C57BL/6 mouse. Administration of erastin into the mice reduced the size of Panc02 parental tumors by $53 \%$, compared with vehicle-treated tumors at day 14 (Figs. S8H and $8 \mathrm{I})$. In agreement with in vitro results, BCAT2 protected Panc02 cancer cells against erastin-induced reduction in tumor growth by twofold (Figs. S8H and 8I). To avoid the off-target action of erastin, we also performed the rescue experiments using $\mathrm{N}$-acetyl-cysteine (NAC, an antioxidant ferroptosis inhibitor) and found that the protective role of BCAT2 on erastin-induced tumor growth inhibition was significantly abolished in the presence of NAC (Figs. S8H and $8 \mathrm{I}$ ). These results indicated that overexpression BCAT2 rescues erastin-induced tumor inhibition.

To further elucidate the functional role of BCAT2 in ferroptosis, two stable BCAT2-knockdown cell clones (BCAT2 shRNA1 and shRNA2) were established with high silencing efficiency (up to $60 \%$ silencing) confirmed by western blotting (Fig. S11A). Compared to the parental cancer cells, BCAT2-knockdown cells showed smaller mitochondria morphology with more condensed mitochondrial membrane density, and reduced mitochondria crista, which are typical morphological features of ferroptosis (Fig. S11B). Furthermore, knockdown of BCAT2significantly increased MDA production (Figs. $2 \mathrm{H}$ and S11D) and GSH depletion (Figs. 2I and S11E) in Aspc-1 and HepG2 cells in the presence of erastin, sorafenib, and sulfasalazine, but had no effect on accumulation of free cellular iron (Figs. 2G and S11C). Moreover, BCAT2 knockdown decreased the level of intracellular glutamate (Figs. 2J and S11F), the glutamate release (Figs. 2K and S11G) as well as cell viability (Figs. $2 \mathrm{~L}$ and $\mathrm{S11H}$ ) in the presence of erastin, sorafenib, or sulfasalazine. Treated parental and BCAT2 knockdown Aspc-1 and HepG2 cells with RSL3 and BSO displayed the similar results (Fig. S12). Accordingly, the colony-formation capability got inhibited in BCAT2 silencing cells (Fig. S11I). Knockdown of BCAT2 did not affect the SLC7A11 and GPX4 protein expression level in Aspc-1 and HepG2 cells (Fig. S13A). These results demonstrated that BCAT2 knockdown could partly induce ferroptosis.

\section{BCAT2 participates in the mechanisms for sulfasalazine synergizing with sorafenib to induce ferroptosis}

As sorafenib or sulfasalazine suppressed BCAT2 knockdown in a similar manner, we hypothesized that sorafenib or sulfasalazine may have synergistic effect in inducing ferroptosis. We first investigated the role of combining sorafenib and sulfasalazine in BCAT2 expression. Intriguingly, we found that combination of sorafenib and sulfasalazine dramatically inhibited BCAT2 expression in Aspc-1 and HepG2 cells (Fig. 3A), in a comparable pattern to sorafenib or sulfasalazine alone, together with BCAT2 shRNA (Figs. 2M and S11J). Sorafenib and sulfasalazine also exhibited a synergistic effect in increasing the cell death (Fig. 3B) and MDA production, which can be rescued in the presence of ferrostatin-1 (Fig. 3C), suppressing the intracellular glutamate level (Fig. 3D), glutamate release 


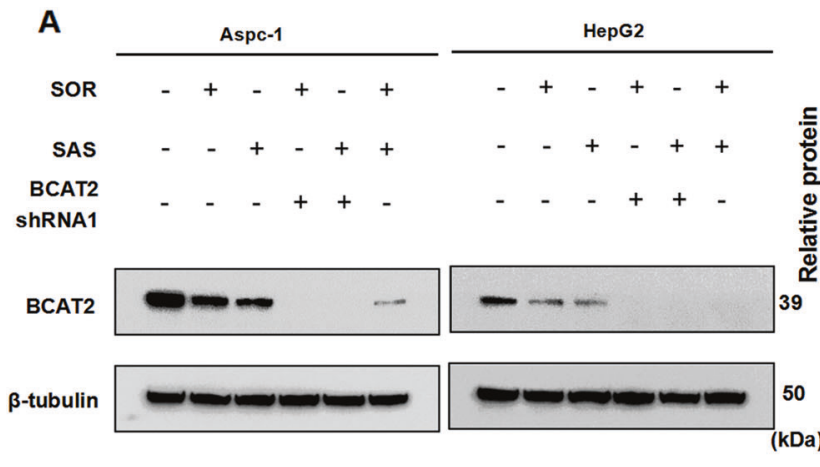

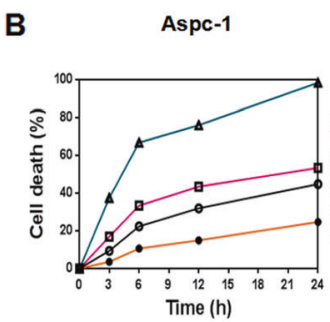

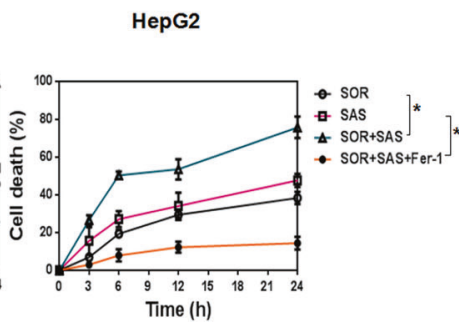

C

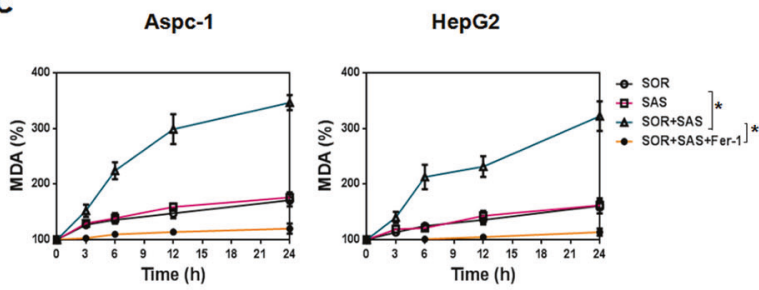

Fig. 3 BCAT2 participates in the mechanisms for sulfasalazine synergizing with sorafenib to induce ferroptosis in vitro. A Western blot analysis of the protein expression levels of BCAT2 in BCAT2 knockdown and parental Aspc-1 and HepG2 cells treated with or without sorafenib $(10$ or $5 \mu \mathrm{mol} / \mathrm{L})$, sulfasalazine $(2$ or $1 \mathrm{mmol} / \mathrm{L})$, or sorafenib $(10$ or $5 \mu \mathrm{mol} / \mathrm{L})+$ sulfasalazine $(2$ or $1 \mathrm{mmol} / \mathrm{L})$. $\beta$-tubulin expression was detected as a loading control. B Aspc-1 and HepG2 cells were treated with sorafenib $(10$ or $5 \mu \mathrm{mol} / \mathrm{L})$, sulfasalazine ( 2 or $1 \mathrm{mmol} / \mathrm{L})$, or sorafenib $(10$ or $5 \mu \mathrm{mol} / \mathrm{L})+$ sulfasalazine $(2$ or $1 \mathrm{mmol} / \mathrm{L})$ in the absence or presence of ferrostatin- $1(1 \mu \mathrm{mol} / \mathrm{L})$ for the indicated time, and cell death was analyzed by CCK8 kit. C Aspc1 and HepG2 cells were treated with sorafenib (10 or $5 \mu \mathrm{mol} / \mathrm{L}$ ),

(Fig. 3E), and expression level of GPX4 (Fig. S13B). All these results support our hypothesis that the effect of sorafenib and sulfasalazine on ferroptosis partially through regulating BCAT2 expression.

We next investigated whether sorafenib and sulfasalazine have synergistic anticancer effect in vivo. Administration of sorafenib and sulfasalazine reduced the size of Panc02 subcutaneous tumors in C57BL/6 mice by $9.63 \%$ and $13.5 \%$, respectively, and the combination therapy further reduced the size by $81.39 \%$, compared with vehicletreated tumors at day 14 (Fig. 4A-C). Since orthotopic xenograft models are considered superior to the subcutaneous tumor models in terms of replicating the tumor microenvironment and predicting of drug efficacy, we would like to check whether induction of ferroptosis by sulfasalazine also enhances the anticancer activity of
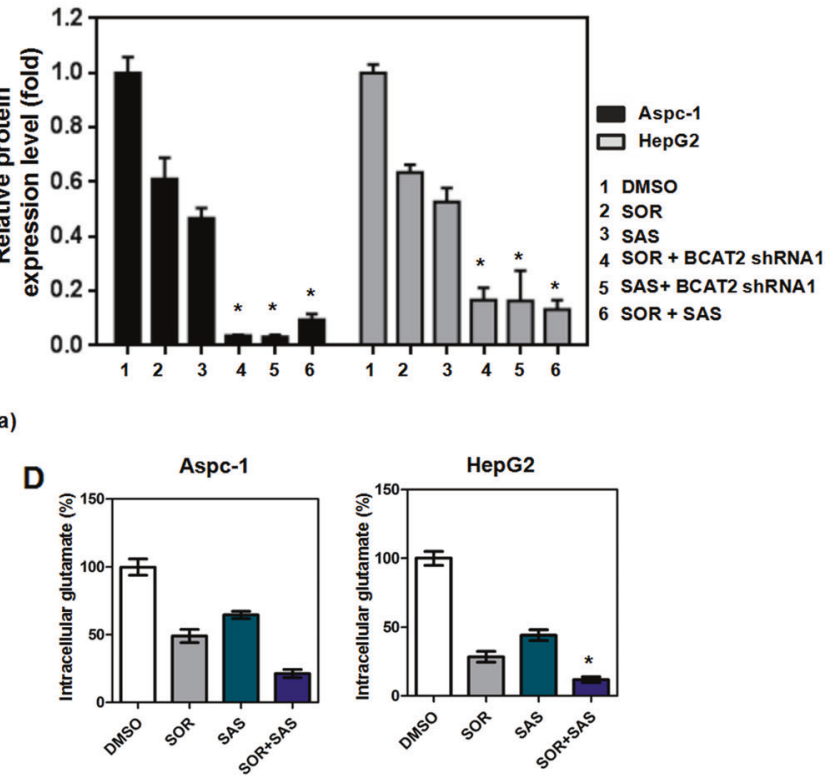

E
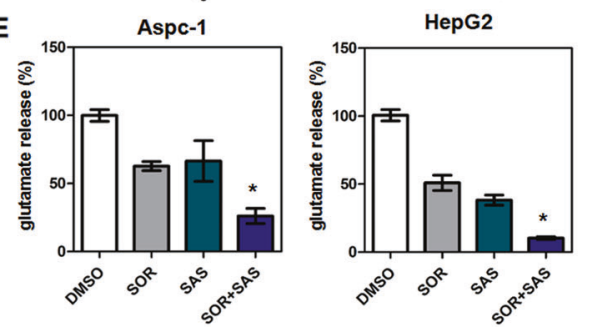

sulfasalazine $(2$ or $1 \mathrm{mmol} / \mathrm{L})$, or sorafenib $(10$ or $5 \mu \mathrm{mol} / \mathrm{L})+$ sulfasalazine $(2$ or $1 \mathrm{mmol} / \mathrm{L})$ in the absence or presence of ferrostatin-1 $(1 \mu \mathrm{mol} / \mathrm{L})$. The relative levels of MDA were assayed. D, E Aspc-1 and HepG2 cells were treated with DMSO (control), sorafenib (10 or $5 \mu \mathrm{mol} / \mathrm{L})$, sulfasalazine $(2$ or $1 \mathrm{mmol} / \mathrm{L})$, or sorafenib (10 or $5 \mu \mathrm{mol} / \mathrm{L})+$ sulfasalazine $(2$ or $1 \mathrm{mmol} / \mathrm{L}$ ). The relative levels of intracellular glutamate $(\mathbf{D})$ and glutamate release $(\mathbf{E})$ were assayed. MDA represents malondialdehyde; Fer-1 represents ferrostatin-1; SOR represents sorafenib; SAS represents sulfasalazine. Experiments were repeated three times, and the data are expressed as the mean $\pm S E M$. $* p<0.05$ vs. control group. Statistical analysis was performed using Student's $t$ test.

sorafenib in orthotopic HCC models with established mouse $\mathrm{H} 22$ cells in C57BL/6 mice (Fig. 4D). Indeed, sorafenib combined with sulfasalazine significantly reduced the tumor size (Fig. 4E) and prolonged animal survival (Fig. 4F) in orthotopic xenograft tumor. Moreover, combination treatment of sorafenib and sulfasalazine significantly reduced BCAT2 in protein- and mRNA-expression levels (Figs. S14A and S14B), diminished GSH level (Fig. S14C), and augmented MDA level (Fig. S14D) in the orthotopic HCC tissues.

In order to reveal more clinical relevance, we turned to a patient-derived xenografts (PDXs) model, which has been applied to preclinical drug testing in many types of cancers due to its biologically stable, and accurately reflect the patient tumor with regards to histopathology, gene expression, genetic mutations, and therapeutic response (Fig. 4G). 
A

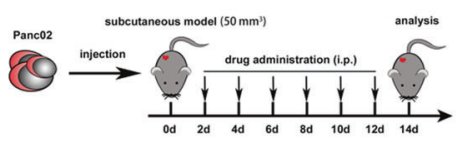

B

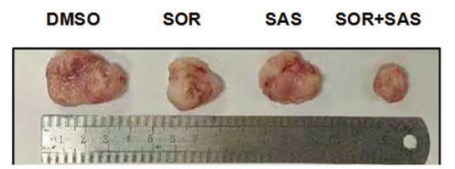

D

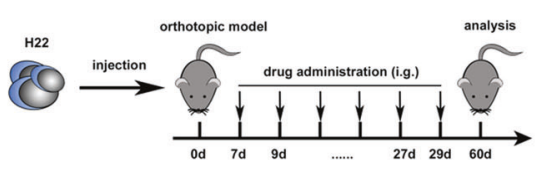

E

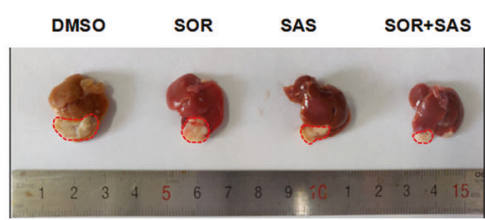

G

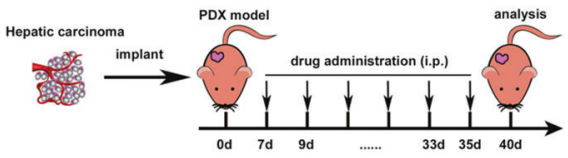

I
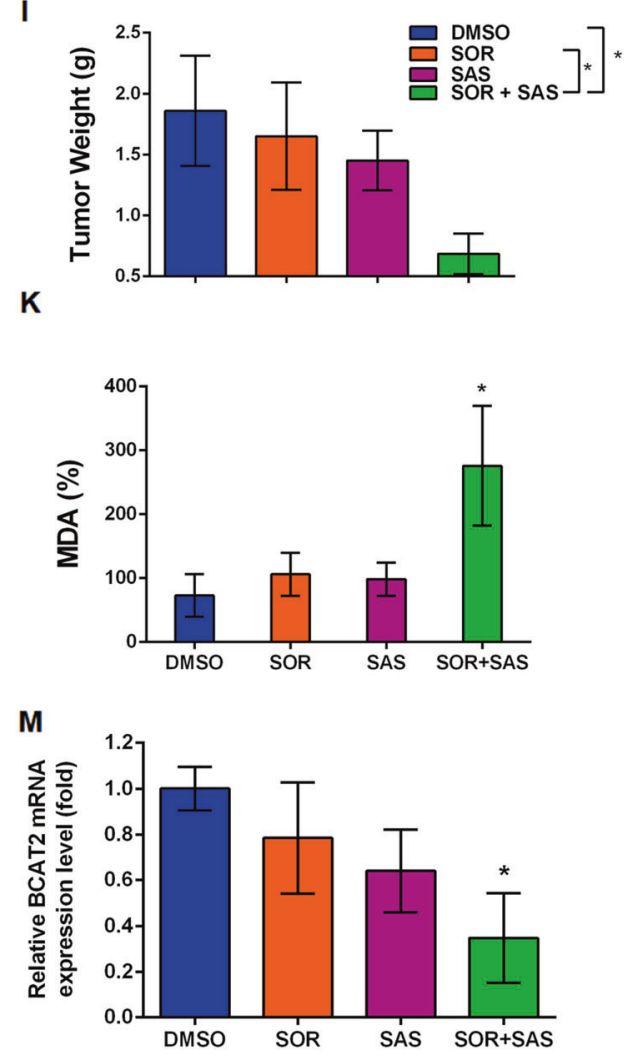

In line with the previous results, sorafenib combined with sulfasalazine significantly reduced the tumor size by $48 \%$ and tumor weight by $63 \%$ in PDX model tumor (Fig. 4H-J).
C

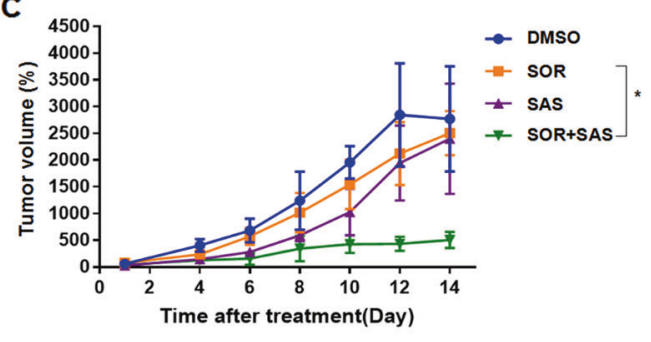

$\mathbf{F}$

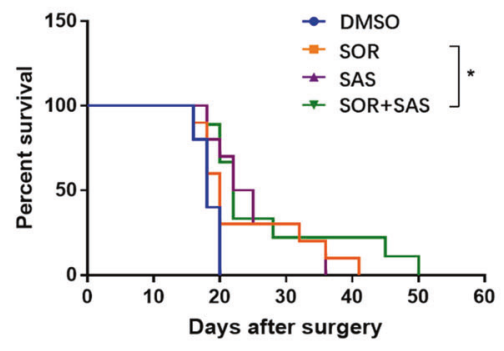

H
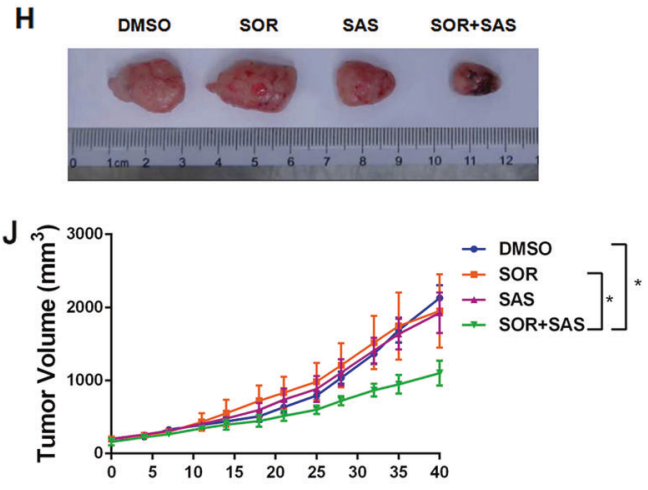

L
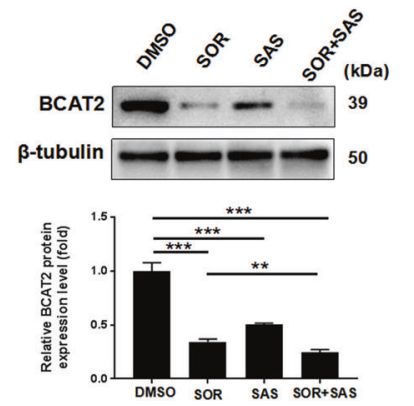

$\mathbf{N}$

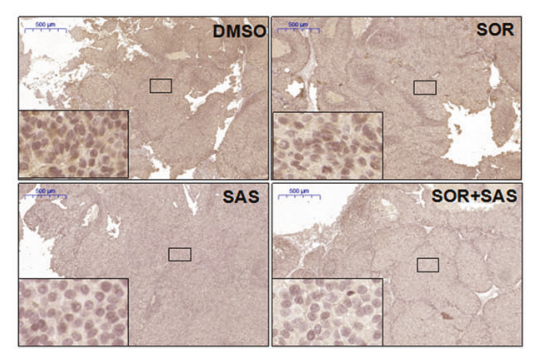

Compared to the control group, sorafenib combined with sulfasalazine significantly augmented locally MDA levels (Fig. 4K). Moreover, there was a significant reduction of 
4 Fig. 4 BCAT2 participates in the mechanisms for sulfasalazine synergizing with sorafenib to induce ferroptosis in vivo. A Schematic representation of the in vivo sorafenib and sulfasalazine combination anticancer effect in Panc02 subcutaneous tumor model. C57BL/ 6 mice were injected subcutaneously with $1 \times 10^{6}$ Panc 02 cancer cells and treated with DMSO (control group), sorafenib (10 mg/kg/i.p., every 2 days), sulfasalazine ( $100 \mathrm{mg} / \mathrm{kg} / \mathrm{i} . p$., every 2 days), or sorafenib $(10 \mathrm{mg} / \mathrm{kg} / \mathrm{i}$. .., every 2 days $)+$ sulfasalazine $(100 \mathrm{mg} / \mathrm{kg} / \mathrm{i} . \mathrm{p}$., every 2 days) for 2 weeks ( $n=5$ mice/group). B Representative photographs of isolated tumor tissues at day 14 after treatment. C Tumor volume was calculated every 2 days. D Schematic representation of the in vivo sorafenib and sulfasalazine combination anticancer effect in $\mathrm{H} 22$ orthotopic xenograft tumor model. $1 \times 10^{6} \mathrm{H} 22$ cells were injected into left lobe of C57BL/6 mice livers and following treated with DMSO (control group), sorafenib (30 mg/kg/i.g., every 2 days), sulfasalazine (300 mg/kg/i.g., every 2 days), or sorafenib $(10 \mathrm{mg} / \mathrm{kg} / \mathrm{i} . g$. ., every 2 days) + sulfasalazine ( $300 \mathrm{mg} / \mathrm{kg} / \mathrm{i} . \mathrm{g}$., every 2 days) for 2 weeks $(n=$ 10 mice/group). E Representative photographs of isolated tumor tissues following various treatments. F Animal survival was calculated every day for 2 months (Kaplan-Meier survival analysis). G Schematic representation of established PDX hepatocellular carcinoma models was treated with DMSO (control group), sorafenib $(10 \mathrm{mg} / \mathrm{kg} / \mathrm{i} . \mathrm{p}$., every 2 days), sulfasalazine ( $100 \mathrm{mg} / \mathrm{kg} / \mathrm{i}$. .., every 2 days), or sorafenib $(10 \mathrm{mg} / \mathrm{kg} / \mathrm{i} . \mathrm{p}$., every 2 days $)+$ sulfasalazine $(100 \mathrm{mg} / \mathrm{kg} / \mathrm{i} . \mathrm{p}$. , every 2 days) for 2 weeks. H Representative photographs of isolated tumor tissues at day 40 after treatment. I Tumor weight of isolated tumor tissues at day 40 after treatment. J Tumor volume was calculated every 3 days. K MDA levels in isolated tumors at day 40 after treatment were assayed. K MDA levels in isolated tumors at day 40 after treatment were assayed. L Western blot analysis of the protein expression level of BCAT2 in isolated tumor tissues at day 40 after treatment. M qRT-PCR analysis of mRNA-expression level of BCAT2 in isolated tumor tissues at day 40 after treatment. N Immunohistochemistry analysis of the expression of BCAT2 in isolated tumor tissues at day 40 after treatment. MDA represents malondialdehyde; i.p. represents intraperitoneal injection; i.g. represents intragastrical administration; SOR represents sorafenib; SAS represents sulfasalazine. Experiments were repeated three times, and the data are expressed as the mean \pm SEM. $* p<0.05$, $* * p<0.01, * * * p<0.001$ vs. control group. Statistical analysis was performed using Student's $t$ test.

BCAT2 in protein and mRNA expression upon combination treatment of sorafenib and sulfasalazine (Fig. 4L-N). These in vivo results further support the in vitro evidence that collective inhibition of the BCAT2 pathway effectively enhances the anticancer activity by induction of ferroptosis.

\section{Discussion}

In this study, we confirmed that erastin, sorafenib, or sulfasalazine activates ferritinophagy and increases cellular labile iron level. High levels of cellular labile iron consequently lead to rapid accumulation of cellular ROS, which is essential for ferroptosis. Interestingly, we found that this ferritinophagy pathway also activates AMPK phosphorylation, which consequently suppresses nuclear translocation of SREBP1, and inhibits the transcription of its direct target gene BCAT2 (Fig. 5). We further revealed BCAT2 as a suppressor of ferroptosis by regulating intracellular glutamate levels. Importantly, the combination of sulfasalazine and sorafenib has synergistic effect in inhibiting BCAT2 expression as well as promoting ferroptotic cancer cell death in vitro and in a couple of animal models including in subcutaneous pancreatic cancer model, orthotopic liver cancer model, as well as PDX hepatic carcinoma model. Of importance, BCAT2 has also shown the potential as a sensitive biomarker to evaluate drug responses in these preclinical cancer models.

The finding that BCAT2 controls the ferroptosis is in accordance with the concept that amino acids play a crucial role in ferroptosis [21]. BCATs are key metabolic proteins catalyzing the reversible transamination of BCAAs to their respective a-ketoacids (BCKAs) and glutamate, responsible for the production of $30 \%$ of de novo brain glutamate $[22,23]$. The metabolism of glutamate is tightly linked to the regulation of ferroptosis $[13,17]$. It is important to note that system $\mathrm{Xc}^{-}$function is regulated by glutamate levels since glutamate is exchanged for cystine in a 1:1 ratio by system $\mathrm{Xc}^{-}$. Accordingly, high extracellular concentrations of glutamate block system $\mathrm{Xc}^{-}$activity, inhibit cystine uptake, and drive ferroptosis [1]. In contrast, high level of intracellular glutamate in our in vitro experiments derived from BCAT2-driven de novo synthesis of glutamate, which may consequently enhance system $\mathrm{Xc}^{-}$activity, boost cystine uptake, and inhibit ferroptosis. This protective effect of BCAT2-intracellular glutamate metabolism is consistent with the fact that there are decreased extracellular brain glutamate levels protected system $\mathrm{Xc}^{-}$knockout mice from neurotoxic injury [24]. Moreover, our experiments confirmed that the expression level of BCAT2 is regulated by AMPK-SREBP1 signaling pathway, which is supported by previous finding that genomic deletion of BCAT2 confers collateral lethality in pancreatic cancer [18]. As a critical sensor of metabolic stress, the role of AMPK in ferroptosis is context-dependent. Song et al. reported AMPK-promoted cancer cells ferroptosis by mediating BECN1 phosphorylation and BECN1-SLC7A11 complex formation [19], which is consistent with our finding that ferroptosis inhibitors downregulate BCAT2 expression through activating AMPK. It is very interesting to notice the very recent report that energy-stress-mediated AMPK activation inhibits ferroptosis [25]. The discrepancy role of AMPK in ferroptosis may be ascribed to the basal AMPK activity of the tested cell lines and the environmental context. Therefore, the potential role of AMPK in cancer ferroptosis deserves deeper investigation and also provides a prime therapeutic strategy for tumor patients.

BCAAs are nitrogen donors for the synthesis of not only glutamate but also glutamine, but the role of glutamine in ferroptosis is complex. Glutamine is degraded through its specific metabolism, glutaminolysis. When glutaminolysis 
Fig. 5 BCAT2 regulates ferroptotic cell death in cancer cells. Ferroptosis inducers (erastin, sorafenib, or sulfasalazine) activate ferritinophagy and AMPK phosphorylation, which consequently suppresses nuclear translocation of SREBP1, and inhibits the transcription of its direct target gene BCAT2. BCAT2 is a suppressor of ferroptosis by regulating intracellular glutamate levels.

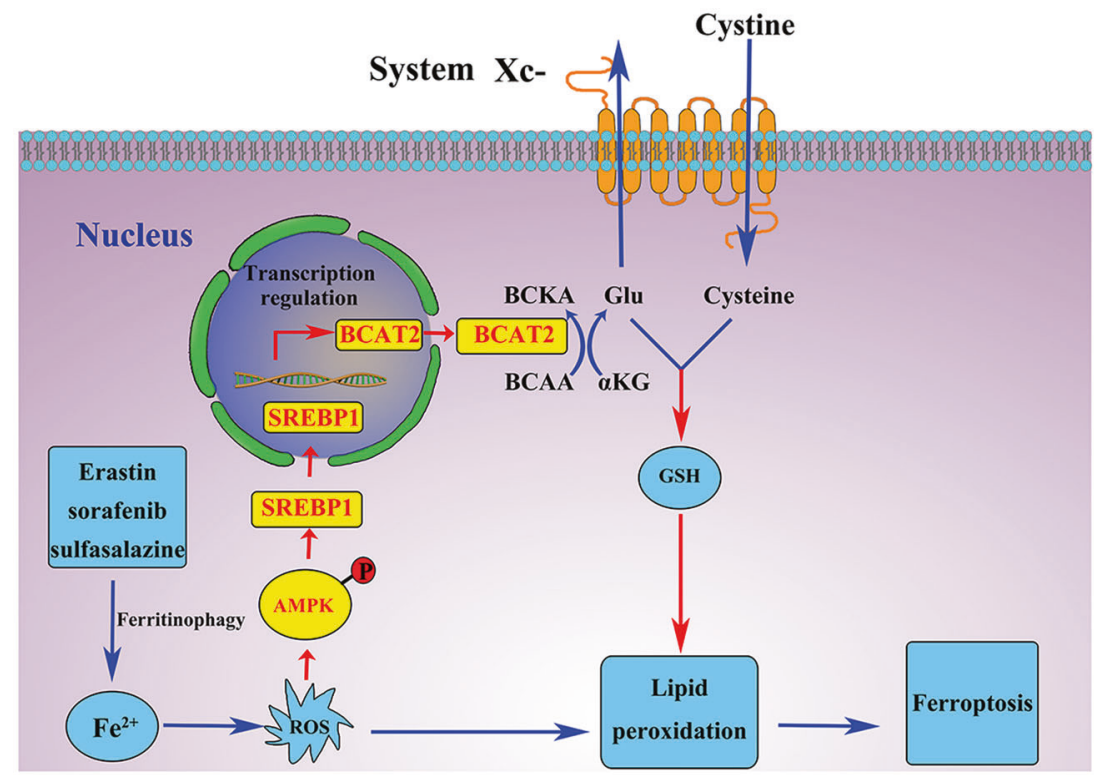

is inhibited or glutamine is depleted, cystine starvation and blockage of cystine import fail to induce ROS accumulation, lipid peroxidation, and ferroptosis, indicating that glutaminolysis fuels ferroptosis. In line with this observation, a-ketoglutarate (a-KG), another product of glutaminolysis besides glutamine, can replace glutamine to induce ferroptosis [17]. We speculate that BCATs catalyze BCAAs-BCKAs shuttle for the synthesis of glutamate, leading to a reduction of intracellular level of a-KG, which may be another reason to induce ferroptosis. Knockdown BCAT2 has no effect on the expression of SLC7A11. Therefore, the exact relationship between BCAT2 and system $\mathrm{Xc}^{-}$needs deeper investigation in the future work.

Liver cancer is the third leading cause of cancer deaths worldwide, and standard chemotherapy has not been effective in most patients with liver cancer, doctors have been looking at targeted therapies. Sorafenib is the only multikinase inhibitor as the first-line treatment proven to prolong overall survival of unresectable HCC [26]. However, the overall survival in patients from the Asia-Pacific region taking sorafenib was just 6.5 months with low response rate of $2 \%$. Lenvatinib is recently approved as an alternate multikinase agent for advanced HCC if sorafenib stops working, but its overall survival superiority over sorafenib was not achieved in a recent phase- 3 clinical study. In this study we found sulfasalazine alone, or in combination with sorafenib, function in ferroptosisinducing therapies. These findings are consequential since sulfasalazine is an anti-inflammatory drug, which has already been used extensively in chronic, long-term therapy of inflammatory bowel disease, guaranteeing its safety profiles both in adults and children [27]. Based on our results, sulfasalazine might be a potential new treatment option for advanced liver cancer, as well as other unresectable cancer types. Due to the expression changes in the treatment, BCAT2 may be one of the most sensitive targets and its expression can be useful as a marker predicting response to sorafenib and sulfasalazine combination treatment. However, this hypothesis should be evaluated in patient data.

Taken together, our data demonstrate that inhibiting intracellular glutamate synthesis could serve as a good strategy for inducing ferroptosis in cancer contexts. This is supported by our finding that sulfasalazine collaborates with sorafenib to downregulate BCAT2 and consequently intracellular glutamate. Our work also suggests a mechanism for cell lethality involving the regulation of de novo synthesis of glutamate as crucial process in liver cancer cells. We suggest that the protein or mRNA level of BCAT2 may be used to predict the responsiveness of cancer cells to future ferroptosis-inducing therapies. We also propose that highly specific BCAT2 inhibitors could provide an effective therapy for a meaningful fraction of cancer patients.

\section{Materials and methods}

\section{Cell culture and reagents}

Aspc-1, HepG2, Panc02, and H22 cell lines were obtained from the KeyGEN Biotechnology Company (China). HT1080 and SW480 were obtained from the FuHeng BioLogy Company (China). HT1080 cancer cells were cultured in Eagle's Minimum Essential Medium supplemented with 10\% fetal bovine serum (FBS), glutamine $(2 \mathrm{mM})$, penicillin $(100 \mathrm{U} / \mathrm{ml})$, and streptomycin $(0.1 \mathrm{mg} / \mathrm{ml})$. SW480, Aspc-1, 
Table 1 Sequences of primers used for qRT-PCR.

\begin{tabular}{lll}
\hline Name & Direction & Sequence $\left(5^{\prime}-3^{\prime}\right)$ \\
\hline BCAT2-human & Forward & CTCTGGGGCAGCTGTTTGA \\
& Reverse & ATAACACCATTCAGCGGGGG \\
BCAT2-mouse & Forward & AAAGCATACAAAGGTGGAGACC \\
& Reverse & CGTAGAGGCTCGTTCCGTTG \\
BCAT1 & Forward & GTGGAGTGGTCCTCAGAGTTT \\
& Reverse & AGCCAGGGTGCAATGACAG \\
GADPH & Forward & TGGGGAAGGTGAAGGTCGG \\
& Reverse & CTGGAAGATGGTGATGGGA \\
\hline
\end{tabular}

HepG2, Panc02, and H22 were cultured in high Dulbecco's Modified Eagle's Medium (DMEM) supplemented with $10 \%$ FBS, L-glutamine $(4 \mathrm{mM})$, and penicillin $(100 \mathrm{U} / \mathrm{ml})$ and streptomycin $(0.1 \mathrm{mg} / \mathrm{ml})$. All cell lines were maintained in a humidified atmosphere containing $5 \% \quad \mathrm{CO}_{2}$ at $37^{\circ} \mathrm{C}$ and tested for mycoplasma prior to the commencement of experiments. Unless otherwise indicated, cell culture medium was changed every 3 days, and cells were passaged using $0.05 \%$ trypsin/EDTA. Erastin (\#HY-15763), sorafenib (\#HY10201), sulfasalazine (\#HY-14655), DON (\#HY-108357), RSL3 (\#HY-100218A), L-Buthionine-(S,R)-sulfoximine (BSO, \#HY-106376A), ferrostatin-1 (\#HY-100579), ZVADFMK (\#HY-16658), AICAR (\#HY-13417), BafA1 (\#HY-100558), N-Acetylcysteine (\#HY-B0215), and Necrosulfonamide (\#HY-100573) were purchased from MedChemExpress (USA). Compound C (\#ab120843) was purchased from Abcam. Deferoxamine mesylate (\#D9533) was purchased from Sigma-Aldrich.

\section{CRISPRa screen}

HepG2 cells were infected with lentivirus-encoding Cas9VPR enzyme and selected with $2 \mu \mathrm{g} / \mathrm{ml}$ puromycin. Briefly, $4.343 \times 10^{7}$ cells were infected with human CRISPRa sgRNA lentivirus library targeting 2320 genes with about 13030 sgRNA at a low MOI (0.3). After $48 \mathrm{~h}$, the infected cells were selected with $800-\mu \mathrm{g} / \mathrm{ml} \mathrm{G} 418$ for $72 \mathrm{~h}$. Cells were equally split into two samples (at least $1.3 \times 10^{7} /$ sample). One sample was treated with $10-\mu \mathrm{M}$ erastin for $16 \mathrm{~h}$ and changed back to DMEM once in 3 days for three rounds compared with another untreated sample as control. Genomic DNA was extracted, and sgRNA was amplified by PCR. The resulting PCR products were sequenced by Illumina Hiseq 4000 and evaluated based on the known sgRNA targets sequence.

\section{Cell viability assay}

Tumor cells were collected and seeded into 96-well plates. After adhesion, cells were treated with the different ferroptosis inducers or inhibitors. To determine the effect of treatment on cell viability, the Cell Counting Kit-8 (CCK8, \#CA1210, Solarbio) was carried out according to the manufacturer's instructions. Absorbance at wavelengths of $450 \mathrm{~nm}$ was measured. The percentage difference in reduction between treated and control cells was calculated. After calculation, the viability of control cells was $100 \%$ and all others were normalized to control and shown as relative cell viability $(\%)$.

\section{Western blot assay}

Protein was quantified using the bicinchoninic acid (BCA) assay (Thermo Fisher Scientific, \#23225). Western blotting assay was performed as described previously [28]. Antibodies were as follows: anti-human BCAT2 (CST, \#9432S, 1:1000), anti-human BCAT1 (Abcam, \#ab197941, 1:1000), anti-human Phospho-AMPKo (Thr172) (CST, \#2535, 1:1000), anti-human AMPK (CST, \#5831, 1:1000), antihuman SREBP1 (Santa Cruz, \#SC-13551, 1:200), antihuman NCOA4 (Abcam, \#ab86707, 1:1000), anti-human ATG7 (CST, \#8558, 1:1000), anti-human SLC7A11 (Abclonal, \#A13685, 1:1000), anti-human GPX4 (Abcam, \#ab41787, 1:1000), and $\beta$-tubulin (Abcam, \#ab6046, 1:1000). Secondary antibody (either anti-rabbit or antimouse) was purchased from Boster Biotechnology Company (China). The blots were analyzed using the software ImageJ (Version 1.80, NIH, USA).

\section{Quatitive real-time polymerase chain reaction assay (qRT-PCR)}

Total RNA was extracted using TRIzol (Invitrogen) according to the manufacturer's instructions. RevertAid First-Strand cDNA Synthesis Kit (Thermo, Waltham, MA, USA) was performed for reverse transcription according to the manufacturer's specification. Subsequently, SYBR Green-based real-time PCR was performed in triplicate using SYBR Green master mix (Thermo Fisher Scientific) on an Applied Biosystems StepOnePlus real-time PCR machine (Thermo Fisher Scientifc). For analysis, the threshold cycle $(\mathrm{Ct})$ values for each gene were normalized to expression levels of GAPDH. Analysis was performed using the Bio-Rad CFX Manager software. The primers, which were synthesized and desalted from Sigma-Aldrich, are shown in Table 1.

\section{Chromatin immunoprecipitation (ChIP)}

ChIP was performed according to the protocol of the ChIP assay kit. Briefly, cells were pretreated erastin, sorafenib, and sulfasalazine, respectively, and then cross-linked in $3.7 \%$ formaldehyde for $15 \mathrm{~min}$, quenched with glycine for 5 min, and lysed with the SDS lysis buffer. Chromatin was 
Table 2 Sequences of shRNAs.

\begin{tabular}{ll}
\hline Name & Sequence $\left(5^{\prime}-3^{\prime}\right)$ \\
\hline BCAT2-shRNA1 & CCGGTGAAGTGCAATACGAAATAA ACTCGAGTTTATTTCGTATTGCACTTCATTTTTG \\
BCAT2-shRNA2 & CCGGGT GCACCGAATCCTGTACAAACTCGAGTTTGTACAGGATTCGGTGCACTTTTTG \\
NCOA4-shRNA1 & CCGGTCAGCAGCTCTACTCGTTATTCTCGAGAATAACGAGTAGAGCTGCTGATTTTTG \\
NCOA4-shRNA2 & CCGGTGAACAGGTGGACCTTATTTACTCGAGTAAATAAGGTCCACCTGTTCATTTTTG \\
ATG7-shRNA1 & CCGGGCCTGCTGAGGAGCTCTCCATCTCGAGATGGAGAGCTCCTCAGCAGGCTTTTT \\
ATG7-shRNA2 & CCGGCCCAGCTATTGGAACACTGTACTCGAGTACAGTGTTCCAATAGCTGGGTTTTT \\
GPX4-shRNA1 & GATCGTGGATGAAGATCCAACCCAACTCGAGTTGGGTTGGATCTTCATCCACTTTTTG \\
GPX4-shRNA2 & GATCGCACATGGTTAACCTGGACAACTCGAGTTGTCCAGGTTAACCATGTGCTTTTTG \\
SREBP1-shRNA1 & CCGGGCCATCGACTACATTCGCTTTCTCGAGAAAGCGAATGTAGTCGATGGCTTTTT \\
SREBP1-shRNA2 & CCGGGCTGAATAAATCTGCTGTCTTCTCGAGAAGACAGCAGATTTATTCAGCTTTTT \\
\hline
\end{tabular}

sheared by sonication, and lysates were precleared with salmon sperm DNA/protein A agarose slurry for $1 \mathrm{~h}$ and incubated with rabbit IgG (Santa Cruz) or SREBP1 antibody in the presence of protein A agarose beads overnight. After sequential washes of the agarose beads and eluted, the elutes were heated at $65^{\circ} \mathrm{C}$ for $4 \mathrm{~h}$ to reverse the crosslinking and treated with RNase A for $30 \mathrm{~min}$ at $37^{\circ} \mathrm{C}$, followed by treatment with proteinase $\mathrm{K}$ for $1 \mathrm{~h}$ at $45^{\circ} \mathrm{C}$ to remove RNA and protein. DNA was recovered, eluted, and then assayed using PCR. The ChIP primers were purchased from Qiagen (EpiTect ChIP PCR assay) and used for qPCR analysis: BCAT2.

\section{CRISPR-Cas9 assay}

Lentivirus carrying SREBP1 sgRNA and control sgRNA was purchased from GENECHEM. HepG2 SREBP1 KO cell line was generated by lentivirus infection and puromycin selection according to the manufacturer's instructions. The target sequence of SREBP1 is CGGGTACATCT TCAATGGAG and the control CRISPR sequence is CGC TTCCGCGGCCCGTTCAA.

\section{RNAi and gene transfection}

Cancer cells were seeded in six-well plates at a density of $1 \times 10^{5}$ cells/well to achieve a confluence of $70-80 \%$ overnight. To generate BCAT2 and SREBP1 knockdown cells, cells were transfected with $10 \mathrm{nM}$ of shRNA against BCAT2 and SREBP1 and negative control shRNA (Suzhou Ribo Life Science CO., Ltd, China), respectively. Transfection was performed with Lipofectamine 2000 (Invitrogen) according to the manufacturer's instructions. The specific shRNA sequences are listed in Table 2.

For establishing the stable sh-BCAT2 cancer cells, the lentiviral packaging kit was purchased from Open GeneCopoeia. Lentivirus carrying BCAT2-shRNA was packaged in $293 \mathrm{~T}$ cells and concentrated from the supernatant, as instructed by the manufacturer's manual. Stable cell lines were established by infecting lentivirus into cancer cells followed by puromycin $(2 \mu \mathrm{g} / \mathrm{ml})$ selection for $10-14$ days. These established stable cell lines were maintained in DMEM containing $10 \%$ FBS and puromycin $(0.75 \mu \mathrm{g} / \mathrm{ml})$ for further experiments.

\section{BCAT2 overexpression experiment}

The mammalian expression plasmid pLVx-BCAT2-Flag (FC-324) was purchased from the Fubio Company (Suzhou, China). Cells were transfected with the stated constructs according to the manufacturer's instructions (Invitrogen, China). Cancer cells were seeded in a 96-well dish at a density of 2000 cells/well. The following day, cells were infected with the vector described above. Cells were infected at an M.O.I. of 10 in media containing $8-\mu \mathrm{g} / \mathrm{mL}$ polybrene and the fluid was changed to fresh normal medium after $24 \mathrm{~h}$. Three days after infection, medium containing $2-\mu \mathrm{g} / \mathrm{mL}$ puromycin was used to screen and select BCAT2-overexpressing cells. After $24 \mathrm{~h}$, cells were treated with various agents for further use.

\section{Iron assay}

Intracellular ferrous iron level was determined using the iron assay kit (Abcam, ab83366) according to the manufacturer's instructions.

\section{Lipid peroxidation assay}

The relative MDA concentration in cell or tumor lysates was assessed using a Lipid Peroxidation (MDA) Assay Kit (Abcam, \#ab118970) according to the manufacturer's instructions. Briefly, MDA in the sample reacts with thiobarbituric acid (TBA) to generate a MDA-TBA adduct. The MDA-TBA adduct can be quantified colorimetrically (OD = 532 nm). C11-BODIPY dye (Thermo Fisher Scientific) was 
used to detect lipid peroxidation in cells. Oxidation of the polyunsaturated butadienyl portion of the dye results in a shift of the fluorescence emission peak from $\sim 590$ to $\sim 510 \mathrm{~nm}$.

\section{Glutathione (GSH) assay}

The relative GSH concentration in cells was assessed using a GSH/GSSG Ratio Detection Assay Kit (Abcam, \#ab205811) according to the manufacturer's instructions. Briefly, whole cell was diluted to 1:80 for GSH analysis, serial dilution of GSH and GSSG stock standards were prepared as standards. A one-step fluorimetric reaction of samples with respective assay buffer and probes was incubated for $30 \mathrm{~min}$. The yellow product (5-thio-2-nitrobenzoic acid) was measured spectrophotometrically at $412 \mathrm{~nm}$.

\section{Glutamate release assay}

The relative release of glutamate from cells into the extracellular medium was detected using an Amplex Red glutamate release assay kit (Thermo Fisher Scientific). Glutamate release was first normalized to the total cell number determined with the CCK8 kit at the end of the experiment, and then values were expressed as a percentage of no-treatment controls.

\section{Intracellular glutamate assay}

The relative intracellular glutamate was detected using a Glutamate Assay Kit (Abcam, \#ab83389) according to the manufacturer's instructions. The intracellular glutamate first normalized to the total cell number determined with the CCK8 kit at the end of the experiment, and then values were expressed as a percentage of no-treatment controls.

\section{TEM imaging}

To observe the subcellular structure, cancer cells treated with various agents were harvested and fixed with $2.5 \%$ glutaraldehyde in 0.1-M sodium cacodylate buffer for $24 \mathrm{~h}$, and subsequently fixed in $1 \%$ osmium tetroxide for $2 \mathrm{~h}$. Specimens were dehydrated in a graded series of acetone and embedded in epoxy resin. After ultramicrotomy, ultrathin sections were stained with uranyl acetate for $15 \mathrm{~min}$ and modified with lead citrate for $5 \mathrm{~min}$. Finally, the subcellular structure was observed by TEM using a JEOL JEM2100 microscope (JEOL Ltd. Japan).

\section{Colony-forming assay}

Cell growth of shRNA-treated cell lines was assayed through crystal violet staining. For colony-formation assays, 2000 cells were seeded in six-well plates for 14 days. Cells were fixed with $80 \%$ methanol and stained with crystal violet solution overnight. All experiments were performed in triplicate.

\section{Immunofluorescence}

HepG2 cells stably expressing GFP-LC3 were grown on glass cover slips in a six-well plate. After $24 \mathrm{~h}$, cells were treated with various agents for $24 \mathrm{~h}$. The cells were then washed with PBS and fixed in $3.7 \%$ paraformaldehyde for $10 \mathrm{~min}$ at $37^{\circ} \mathrm{C}$. The number of GFP-LC3 dots was then detected using a confocal fluorescence microscope.

\section{Immunohistochemistry analysis}

Paraffin-embedded tissues were sectioned for immunohistochemical (IHC) analysis. For IHC, samples were fixed in $10 \%$ formalin and embedded in paraffin wax. Next, 3-mm sections were cut from the paraffin blocks for IHC analysis. The sections were stained with mouse rabbit-BCAT2 (Abcam, \#ab95976, 1:200) at $4{ }^{\circ} \mathrm{C}$ overnight. All the sections were cover slipped with neutral balsam and viewed under an Olympus microscope and analyzed using ImageJ software. The final result for each case was the average score of all visual fields.

\section{Xenograft tumor models}

Animal studies were approved by the Committee on the Use of Live Animals for Teaching and Research of the Jiangsu University. Female C57BL/6 mice (purchased from The Compare Medicine Center, Yangzhou University, China), age 4 weeks, were health checked daily throughout the experiment and kept on a regular 12-h light and dark cycle with normal diet in a pathogen-free barrier facility.

$1 \times 10^{6}$ BCAT2 overexpression and control Panc02 cancer cells were implanted subcutaneously into the right dorsal flanks of C57BL/6 mice (five mice per group), respectively. When the tumors reached a volume of $50-100 \mathrm{~mm}^{3}$, the mice were treated with or without erastin $(40 \mathrm{mg} / \mathrm{kg})$ every 2 days for 2 weeks. Due to the low solubility and poor metabolic stability, erastin was administration by the intratumoral injection. The tumor volume and growth speed were monitored every 2 days until the end point at day 14 .

To investigate the role of combination sorafenib with sulfasalazine inducing ferroptosis, $1 \times 10^{6}$ Panc02 were implanted subcutaneously into the right dorsal flanks of C57BL/6 mice. When the tumors reached a volume of $50-100 \mathrm{~mm}^{3}$, mice were randomly divided into four groups (five mice per group) and treated with DMSO (control), sorafenib $(10 \mathrm{mg} / \mathrm{kg})$, sulfasalazine $(100 \mathrm{mg} / \mathrm{kg})$, or the combination of these drugs at the indicated doses by intraperitoneal injection every 2 days for 2 weeks. The 
tumor volume and growth speed was monitored every 2 days until the end point at day 14 .

To generate orthotopic tumors, forty C57BL/6 mice were surgically implanted with $1 \times 10^{6} \mathrm{H} 22$ cells into left lobe of livers. One week after implantation, mice were randomly allocated into four groups (ten mice per group) and treated with the following agents: (i) DMSO; (ii) sorafenib (30 mg/ $\mathrm{kg})$; (iii) sulfasalazine $(300 \mathrm{mg} / \mathrm{kg})$; or (iii) sorafenib $(30 \mathrm{mg} / \mathrm{kg})+$ sulfasalazine $(300 \mathrm{mg} / \mathrm{kg})$ by intragastrical administration every 2 days for 3 weeks. Animal survival was calculated every day for 2 months. Fresh tumor tissue weight was immediately accessed following harvest.

\section{PDXs and in vivo experiments}

NSG (NOD. Cg-Prkdc $c^{\text {sid }} I 2^{\text {rgtm } / W j l} / \mathrm{SzJ}$ ) mice were purchased from the BEIJING IDMO Co., Ltd. and maintained in Animal Center of Jiangsu University in compliance with the Guide for the Care and Use of Laboratory Animals (NIH Publication No. 85-23, revised 1996). The experimental protocols were approved by the Committee for Ethical Affairs of Jiangsu University (Zhenjiang, China), and the methods were carried out in accordance with the approved guidelines.

Serial passaging of the PDX was carried out by implanting small fragments of the liver tumor subcutaneously into dorsal flanks of NSG mice. Experiments were performed using PDX tumor passages 4 and 5. PDXs mice were randomly allocated into four groups (five mice per group) and treated with the following agents: (i) DMSO; (ii) sorafenib (10 mg/kg); (iii) sulfasalazine $(100 \mathrm{mg} / \mathrm{kg})$; or (iii) sorafenib $(10 \mathrm{mg} / \mathrm{kg})+$ sulfasalazine $(100 \mathrm{mg} / \mathrm{kg})$ by intraperitoneal every 2 days for 28 days. The body weight, tumor volume, and growth speed were monitored every 2 days until the end point at day 40 . Tumor weight was fresh tumor tissues from all the mice. Tumor tissues were stored for MDA assay, qRT-PCR, west blotting, and immunohistochemistry analysis.

\section{Patient selection}

TCGA database: https://tcga.xenahubs.net/download/TCGA. LIHC.sampleMap/HiSeqV2.gz; TCGA. LIHC.sampleMap/ HiSeqV2. HCC gene expression by RNAseq (IlluminaHiSeq percentile) including 390 hepatic carcinoma patient specimens was utilized to further analyze the relationship between BCAT2, GPX4, NCOA4, TP53, BECN1, NRF2, and SLC7A11. High and low groups were defined as above and below the mean, respectively.

\section{Statistics}

All data are presented as the mean \pm standard error of the mean. Linear regression and $\mathrm{F}$ testing were used to determine correlation between BCAT2, GPX4, NCOA4, TP53, BECN1, NRF2, and SLC7A11 in HCC. The significances of differences between groups were analyzed using Student's $t$ tests, one-way analysis of variance (ANOVA) or two-way ANOVA. $P<0.05$ was considered to reflect a statistically significant difference. All the experiments were repeated at least three times.

Acknowledgements This study was supported by grants from the National Natural Science Foundation of China (Grant Numbers 82071984, 81702750, 81670141, 81970145), the Social Development Foundation of Jiangsu Province (Grant Number BE2018691, BK20191223), Key Programme of Jiangsu Commission of Health (K2019024), Young Medical Talents of Jiangsu (Grant Number QNRC2016833), Six talent peals project of Jiangsu Province (Grant Number WSW-039), Six for one project of Jiangsu Province (Grant Number LGY2018093), Project of China International Medical Foundation SKY Medical Imaging (Z-2014-07-1912-18), Social Development Foundation of Zhenjiang City (Grant Numbers SH2018031), Natural Science Foundation of Guangdong Province (2020A1515011465), the Basic Research Project of Shenzhen (Grant Numbers JCYJ20170818164756460, JCY J20180307154700308, JCYJ20170818163844015, JCYJ20180307151420045, and JCYJ20190807151609464), Sun Yat-sen University (20ykzd17), International Collaboration of Science and Technology of Guangdong Province (2020A0505100031), China Postdoctoral Science Foundation (2018M643299), and Sigrid Jusélius foundation in Finalnd.

\section{Compliance with ethical standards}

Conflict of interest The authors declare that they have no conflict of interest.

Publisher's note Springer Nature remains neutral with regard to jurisdictional claims in published maps and institutional affiliations.

Open Access This article is licensed under a Creative Commons Attribution 4.0 International License, which permits use, sharing, adaptation, distribution and reproduction in any medium or format, as long as you give appropriate credit to the original author(s) and the source, provide a link to the Creative Commons license, and indicate if changes were made. The images or other third party material in this article are included in the article's Creative Commons license, unless indicated otherwise in a credit line to the material. If material is not included in the article's Creative Commons license and your intended use is not permitted by statutory regulation or exceeds the permitted use, you will need to obtain permission directly from the copyright holder. To view a copy of this license, visit http://creativecommons. org/licenses/by/4.0/.

\section{References}

1. Dixon SJ, Lemberg KM, Lamprecht MR, Skouta R, Zaitsev EM, Gleason CE, et al. Ferroptosis: an iron-dependent form of nonapoptotic cell death. Cell. 2012;149:1060-72.

2. Dixon SJ. Ferroptosis: bug or feature? Immunological Rev. 2017;277:150-7.

3. Hambright WS, Fonseca RS, Chen L, Na R, Ran Q. Ablation of ferroptosis regulator glutathione peroxidase 4 in forebrain neurons promotes cognitive impairment and neurodegeneration. Redox Biol. 2017;12:8-17. 
4. Friedmann Angeli JP, Schneider M, Proneth B, Tyurina YY, Tyurin VA, Hammond VJ, et al. Inactivation of the ferroptosis regulator Gpx4 triggers acute renal failure in mice. Nat Cell Biol. 2014;16:1180-91.

5. Lu B, Chen XB, Ying MD, He QJ, Cao J, Yang B. The role of ferroptosis in cancer development and treatment response. Front Pharm. 2017;8:992.

6. Xie Y, Hou W, Song X, Yu Y, Huang J, Sun X, et al. Ferroptosis: process and function. Cell Death Differ. 2016;23:369-79.

7. Dixon SJ, Patel DN, Welsch M, Skouta R, Lee ED, Hayano M, et al. Pharmacological inhibition of cystine-glutamate exchange induces endoplasmic reticulum stress and ferroptosis. Elife. 2014; 3:e02523.

8. Ooko E, Saeed ME, Kadioglu O, Sarvi S, Colak M, Elmasaoudi $\mathrm{K}$, et al. Artemisinin derivatives induce iron-dependent cell death (ferroptosis) in tumor cells. Phytomedicine. 2015;22:1045-54.

9. Liu N, Lin X, Huang C. Activation of the reverse transsulfuration pathway through NRF2/CBS confers erastin-induced ferroptosis resistance. Br J Cancer. 2020;122:279-92.

10. Sun X, Ou Z, Xie M, Kang R, Fan Y, Niu X, et al. HSPB1 as a novel regulator of ferroptotic cancer cell death. Oncogene. 2015;34:5617-25.

11. Hasegawa M, Takahashi H, Rajabi H, Alam M, Suzuki Y, Yin L, et al. Functional interactions of the cystine/glutamate antiporter, CD44v and MUC1-C oncoprotein in triple-negative breast cancer cells. Oncotarget. 2016;7:11756-69.

12. Stockwell BR, Friedmann Angeli JP, Bayir H, Bush AI, Conrad M, Dixon SJ, et al. Ferroptosis: a regulated cell death nexus linking metabolism, redox biology, and disease. Cell. 2017;171: 273-85.

13. Angeli JPF, Shah R, Pratt DA, Conrad M. Ferroptosis inhibition: mechanisms and opportunities. Trends Pharm Sci. 2017;38:489-98

14. Sun X, Ou Z, Chen R, Niu X, Chen D, Kang R, et al. Activation of the p62-Keap1-NRF2 pathway protects against ferroptosis in hepatocellular carcinoma cells. Hepatology. 2016;63:173-84.

15. Hou W, Xie Y, Song X, Sun X, Lotze MT, Zeh HJ 3rd, et al. Autophagy promotes ferroptosis by degradation of ferritin. Autophagy. 2016;12:1425-8.

16. Hayano M, Yang WS, Corn CK, Pagano NC, Stockwell BR. Loss of cysteinyl-tRNA synthetase (CARS) induces the transsulfuration pathway and inhibits ferroptosis induced by cystine deprivation. Cell Death Differ. 2016;23:270-8.

17. Gao M, Monian P, Quadri N, Ramasamy R, Jiang X. Glutaminolysis and transferrin regulate ferroptosis. Mol Cell. 2015;59:298-308.

18. Dey P, Baddour J, Muller F, Wu CC, Wang H, Liao WT, et al. Genomic deletion of malic enzyme 2 confers collateral lethality in pancreatic cancer. Nature. 2017;542:119-23.

19. Song X, Zhu S, Chen P, Hou W, Wen Q, Liu J, et al. AMPKmediated BECN1 phosphorylation promotes ferroptosis by directly blocking system Xc(-) activity. Curr Biol. 2018;28:2388-99.e5.

20. Gao M, Monian P, Pan Q, Zhang W, Xiang J, Jiang X. Ferroptosis is an autophagic cell death process. Cell Res. 2016;26:1021-32.

21. Gao M, Jiang X. To eat or not to eat-the metabolic flavor of ferroptosis. Curr Opin Cell Biol. 2018;51:58-64.

22. LaNoue KF, Berkich DA, Conway M, Barber AJ, Hu LY, Taylor $\mathrm{C}$, et al. Role of specific aminotransferases in de novo glutamate synthesis and redox shuttling in the retina. J Neurosci Res. 2001; 66:914-22.

23. Lieth E, LaNoue KF, Berkich DA, Xu B, Ratz M, Taylor C, et al. Nitrogen shuttling between neurons and glial cells during glutamate synthesis. J Neurochem. 2001;76:1712-23.

24. Massie A, Schallier A, Kim SW, Fernando R, Kobayashi S, Beck $\mathrm{H}$, et al. Dopaminergic neurons of system $\mathrm{x}(\mathrm{c})(-)$-deficient mice are highly protected against 6-hydroxydopamine-induced toxicity. FASEB J: Off Publ Federation Am Societies Exp Biol. 2011; 25:1359-69.

25. Lee H, Zandkarimi F, Zhang Y, Meena JK, Kim J, Zhuang L, et al. Energy-stress-mediated AMPK activation inhibits ferroptosis. Nat Cell Biol. 2020;22:225-34.

26. Tovoli F, Ielasi L, Casadei-Gardini A, Granito A, Foschi FG, Rovesti G, et al. Management of adverse events with tailored sorafenib dosing prolongs survival of hepatocellular carcinoma patients. J Hepatol. 2019;71:1175-83.

27. Scaioli E, Sartini A, Liverani E, Digby RJ, Ugolini G, Rosati G, et al. Sulfasalazine in prevention of pouchitis after proctocolectomy with ileal pouch-anal anastomosis for ulcerative colitis. Dig Dis Sci. 2017;62:1016-24.

28. Chen X, Cheng F, Liu Y, Zhang L, Song L, Cai X, et al. Toll-like receptor 2 and Toll-like receptor 4 exhibit distinct regulation of cancer cell stemness mediated by cell death-induced high-mobility group box 1. EBioMedicine. 2019;40:135-50. 